\title{
Component-based Model for Flap Noise Prediction
}

DOI:

10.2514/6.2019-2575

\section{Document Version}

Accepted author manuscript

Link to publication record in Manchester Research Explorer

\section{Citation for published version (APA):}

Jiang, Y., \& Filippone, A. (2019). Component-based Model for Flap Noise Prediction. In AIAA/CEAS Aeroacoustics Conference https://doi.org/10.2514/6.2019-2575

\section{Published in:}

AIAA/CEAS Aeroacoustics Conference

\section{Citing this paper}

Please note that where the full-text provided on Manchester Research Explorer is the Author Accepted Manuscript or Proof version this may differ from the final Published version. If citing, it is advised that you check and use the publisher's definitive version.

\section{General rights}

Copyright and moral rights for the publications made accessible in the Research Explorer are retained by the authors and/or other copyright owners and it is a condition of accessing publications that users recognise and abide by the legal requirements associated with these rights.

\section{Takedown policy}

If you believe that this document breaches copyright please refer to the University of Manchester's Takedown Procedures [http://man.ac.uk/04Y6Bo] or contact uml.scholarlycommunications@manchester.ac.uk providing relevant details, so we can investigate your claim.

\section{OPEN ACCESS}




\title{
Component-based Model for Flap Noise Prediction
}

\author{
Yi Jiang* \\ China Aerodynamics Research and Development Center (CARDC) \\ A Filippone \\ The University of Manchester
}

\begin{abstract}
A component-based model is proposed for flap noise prediction to be used in the context of a comprehensive framework for aircraft noise. The flap noise are decomposed into three distinct acoustic contributions: trailing-edge contribution, low and high frequency side-edge contribution. Except the side-edge noise, the trailing-edge noise is recognized as a comparable contribution in the flap noise. Functions and parameters are derived for the energy conversion efficiency, the normalized spectra and the acoustic directivity. Applications of the model are shown for typical flap noise predictions, which include an isolated Airbus A320 flap, a half-span Fowler flap and a flap in a high-lift wing. It is demonstrated that the model proposed is suitable for aircraft noise prediction, with experimental data well predicted.
\end{abstract}

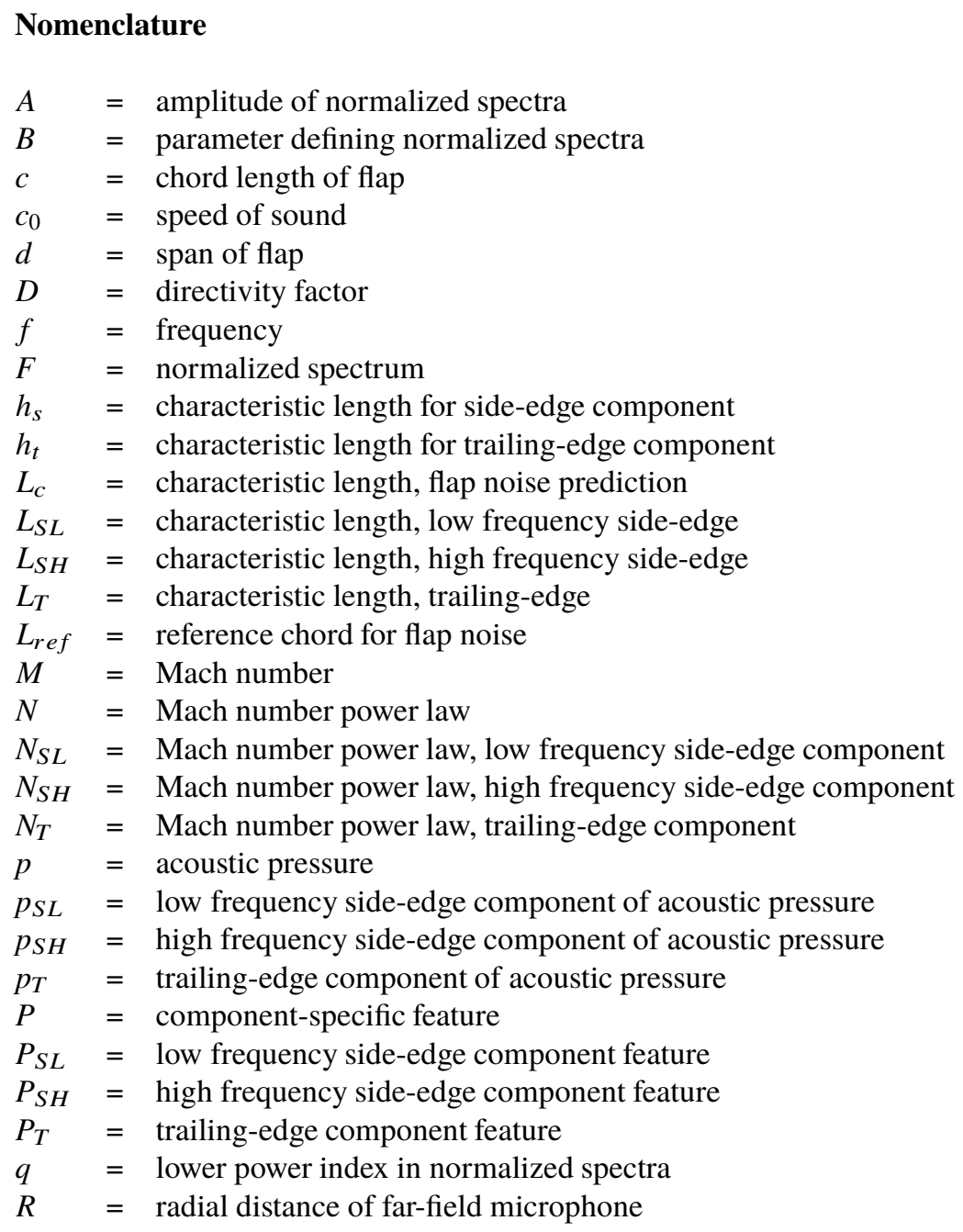

*Computational Aerodynamics Institute, P.O. Box 211, Mianyang 621000, PR China. Email:

${ }^{\dagger}$ School of Mechanical, Aerospace, Civil Engineering, AIAA Senior Member. Email: 


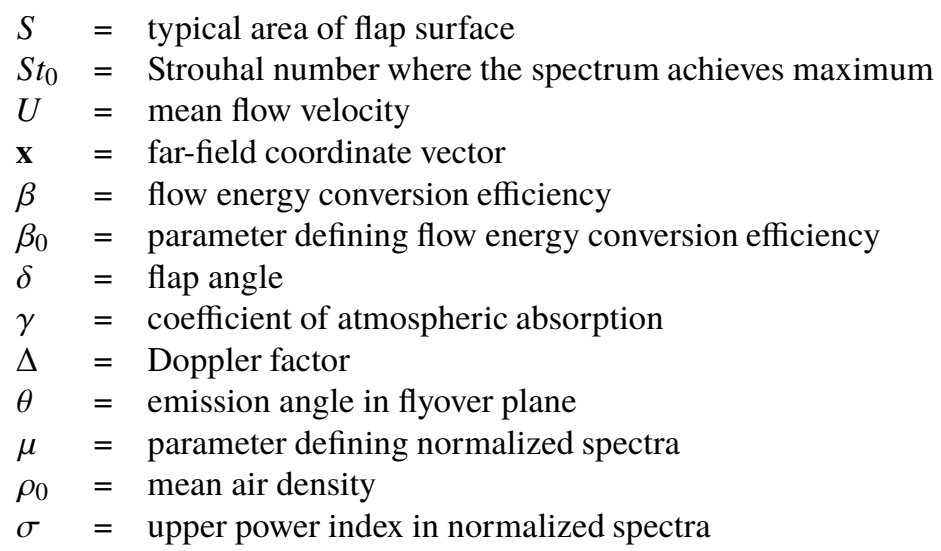

\section{Introduction}

Airframe noise is a major contributor to the overall sound level of an aircraft during final approach and landing [1]. High-lift systems, fully deployed in most approach and landing configurations, include leading edge slats and trailing-edge flaps. These systems show comparable levels of aerodynamically-generated noise [2, 3]. The trailingedge flap system is the subject of this investigation. Specifically, this paper presents a new low-order method to be used within the context of comprehensive aircraft noise prediction of single trajectories, multiple take-off and landings, noise footprints, and more. These applications may require thousands to million of airframe noise calculations, something that cannot be practically achieved with high-order methods (computational fluid dynamics, CFD, and computational aero-acoustics, CAA). Another aspect of the problem is that in many cases the exact geometry of the configuration is unknown, which makes it impossible to produce a sufficiency accurate prediction model using high-order field models.

Therefore, there is a need to improve our noise prediction capabilities with methods that couple some physics with basic geometrical configurations. The approach to be followed, then, is to extract as much information as possible from the physics of a generic configuration, understand the noise production mechanisms, derive low-fidelity models and then apply these models to a chain of noise predictions for an end-to-end engineering process.

Pioneering work in this area was performed by Fink [4], who proposed a simple, entirely empirical model, to predict the noise from airframe components on the basis of a very limited number of parameters. Empirical tools for the prediction of airframe noise have been developed more recently [5, 6] on the basis of large experimental and numerical databases of aircraft noise. These data are available for understanding the basic mechanisms of noise generation and for calibrating low-order prediction models.

A fundamental understanding of the airflow around a flap is required before developing a low-order method for flap noise. Wind-tunnel experiments and CFD are two common tools to investigate the airflow. The vortex roll-up at the flap side-edge and its noise generation mechanisms have been comprehensively recognized from a series of experimental and numerical studies [7-14]. A double vortex structure is mainly responsible for aeroacoustic noise radiation at the flap side-edge. The vortices in the double vortex system are formed by unsteady shear layers shedding from the suction and pressure sides at the side-edge. These vortices eventually merge to a single vortical structure on suction side of the flap. The unsteady shear layers have been recognized as the main mechanisms responsible for flap side-edge noise. Supported by a stability analysis made by Streett et al. [7] and wind tunnel measurements conducted by Choudhari et al. [10], the shear layer instability mechanism contributes to broadband side-edge noise.

While noise from flap side-edges has been the focus of numerous studies, trailing-edge noise from the flap has received less attention. However, trailing-edge noise has been well recognized in several studies [15-21]. Trailing-edge noise should certainly be included in flap noise models. It is worth noting that the focused trailing-edge component here in the flap noise is radiated in the flow condition of high angles of attack caused by deployed high-lift systems. In particular, Brooks \& Schlinker [16] described the trailing-edge noise mechanisms in some detail. At high angles of attack, this noise mechanism is similar to that of a bluff body; the separated flow near the trailing-edge gives way to large-scale separation causing the airfoil to radiate noise. Although the trailing edge component is important, it is usually neglected in empirical prediction of the flap noise. Only addressing the side-edge noise, Guo proposed an empirical model for flap noise prediction [22]. Rossignol developed this model by choosing appropriate parameters for the characteristic velocity and length scales [13]. 
Considering the basic noise mechanisms of flap noise described above, a component-based model can be developed for flap noise prediction. In the component-based model, the flap noise is decomposed into several components. This decomposition is inspired by the component-based concept of Dobrzynski et al. [23], which has been further used to develop a component-based model for airframe noise prediction [13, 22, 24, 25]. Based on a rational decomposition consistent with the flap noise mechanisms, the component-based model in the present study is derived by adopting the scaling laws from the theory of aerodynamic noise generation. This model has a general trend described by the inverse fourth power law of convective amplification [26-30]. The modified power law proposed by Rossignol [13] is employed, since the sixth power law of the Mach number is unsuitable for flap noise prediction.

We propose a noise decomposition into three domains: the low frequency side-edge component, the high frequency side-edge component, and the trailing-edge component. The tailing-edge component is generated by vortex shedding from the flap trailing-edge. The vortices at the side-edges are responsible for the broadband side-edge noise, which is decomposed into the low frequency component and the high frequency component. Rossignol [13] decomposed the broadband side-edge noise into low- and high frequency components to develop an empirical model for flap noise, but the trailing-edge noise component is not considered. After the decomposition, the normalized spectrum, the flow energy conversion efficiency and the directivity factors are fitted on the basis of experimental data. The capability of the model is examined by applying it to the noise prediction for various flaps, including an isolated A320-type flap, a half-span Fowler flap and a flap in a high-lift wing model.

\section{Prediction model}

According to the concept of noise decomposition, the far-field acoustic pressure of a flap shown in Figure 1 can be expressed as

$$
\left\langle p^{2}\right\rangle=\left\langle p_{S L}^{2}\right\rangle+\left\langle p_{S H}^{2}\right\rangle+\left\langle p_{T}^{2}\right\rangle
$$

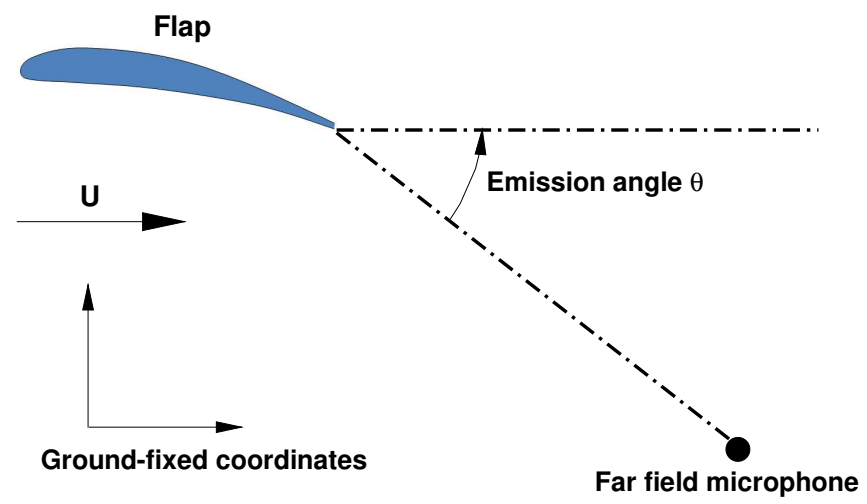

\section{Fig. 1 Illustration and nomenclature of a flap geometry.}

where the subscripts $S L, S H$ and $T$, respectively, indicate the low frequency side-edge component, the high frequency side-edge component, and the trailing-edge component. By starting with the Ffowcs-Williams-Hawking equation [27] and applying scaling laws [24], the acoustic pressure can be written as

$$
\left\langle p^{2}\right\rangle=\frac{\left(\rho_{0} c_{0}^{2}\right)^{2} e^{-\gamma R} S}{R^{2} \Delta^{4}}\left\{P_{S L}+P_{S H}+P_{T}\right\}
$$

where $S=c \cdot d$ is the reference surface area of the flap and $\Delta=1-M \cos \theta$. The features common to all three components have been factored, out and component-specific ones are represented by the quantity $P$, with respective subscripts for the three components. These latter factors are defined by

$$
P=\beta M^{N} D(\theta) F(S t)
$$


The power factor is $N=6$ according to the scaling laws [24]. However, this simple integer is unsuitable for the flap noise prediction. The power is $N_{S L}=5.5$ for low frequency side-edge component and $N_{S H}=6.5$ for high frequency side-edge component. For the trailing-edge component, the power is set to $N_{T}=5.5$, which is in good agreement with the fitted result 5.4 and 5.6, respectively provided by Cozza et al. [31] and Ewert et al. [32], based on CFD solutions and experimental data. To close the prediction model, we also need to formulate the normalized spectrum $F(S t)$, the flow energy conversion efficiency $\beta$ and the directivity factor $D(\theta)$.

\section{A. Normalized spectrum}

The normalized spectra are defined in terms of the Strouhal number $S t$

$$
S t=f L_{c} / U
$$

In Eq. 4, $L_{c}$ is the characteristic length dominated by the noise mechanism. As discussed earlier, the two structures, i.e. vortices rolling from side-edges and the trailing-edge are mainly responsible for the flap noise radiation. Since vortices from trailing-edge are linked to the trailing-edge component of the flap noise, the characteristic length of this component is given by

$$
L_{T}=h_{t} L_{r e f}
$$

where, $L_{r e f}$ is a reference chord length, and $h_{t}$ is a parameter to control the position of the spectral peak. The constraints defining the position of the spectral peak are given in Eqs. 8 and 9 . The quantity $L_{r e f}$ is affected by anything dominating the noise mechanism, such as porous edge and edge geometry. In this model, we choose $L_{r e f}=0.48 \mathrm{~m}$ and $h_{t}=0.12$ for a non-porous and blunt edge. The values of these two parameters are determined to complete the construction of the normalized spectrum. The characteristic lengths of the two components of the side-edge noise are the same, which can be written as

$$
L_{S L}=L_{S H}=h_{s} L_{r e f},
$$

where $h_{s}=0.15$, approximately corresponding to the starting position of the vortices rolling from the side-edge. The $h_{s}$ is also proposed for a non-porous and blunt edge based on experimental data.

With characteristic lengths (5) and (6), the Strouhal number can be calculated for each separate component, then the normalized spectrum is obtained from a general form

$$
F(S t)=A \frac{S t^{\sigma}}{\left(B+S t^{\mu}\right)^{q}}
$$

The spectrum $F(S t)$ achieves a maximum at the Strouhal number

$$
S t=S t_{0}
$$

with two conditions [24],

$$
\begin{aligned}
& A=(q \mu / \sigma)^{q} S t_{0}^{q \mu-\sigma}, \\
& B=\{(\mu q / \sigma)-1\} S t_{0}^{\mu} .
\end{aligned}
$$

Once the empirical constants $q, \mu, \sigma$ and $S t_{0}$ are given by fitting with experimental data, the normalized spectra are determined. These parameters are given in Table 1 An illustration of three normalized spectra is plotted in Figure 2 for the nearly full-scale flap of the Airbus A320 [13], as a function of frequency in a range between $100 \mathrm{~Hz}$ and $10,000 \mathrm{~Hz}$. These spectra are designed to represent the two main sources, i.e. vortices from the trailing-edge and from side-edges.

\section{B. Energy conversion efficiency}

The energy conversion efficiency $\beta$ describes the transition between airflow and noise generation. The conversion efficiency can be derived by matching predictions with experimental data for the three spectral components. Our understanding of the flap noise is that the far-field SPL increases with the deflection angle $\delta$. This basic principle can be validated by experimental data [11, 13]. With this in mind, we propose a model of the conversion efficiency for the low frequency side-edge component and tailing-edge component 
Table 1 Parameters of the normalized spectra.

\begin{tabular}{cccc}
\hline & For $P_{S L}$ & For $P_{S H}$ & For $P_{T}$ \\
\cline { 2 - 4 }$S t_{0}$ & 0.8 & 4.0 & 1.6 \\
$\sigma$ & 4.0 & 3.0 & 5.0 \\
$\mu$ & 2.5 & 1.5 & 2.1 \\
$q$ & 2.6 & 4.2 & 5.2 \\
$A$ & 2.02 & 2188.36 & 938.65 \\
$B$ & 0.36 & 8.80 & 3.18 \\
\hline
\end{tabular}

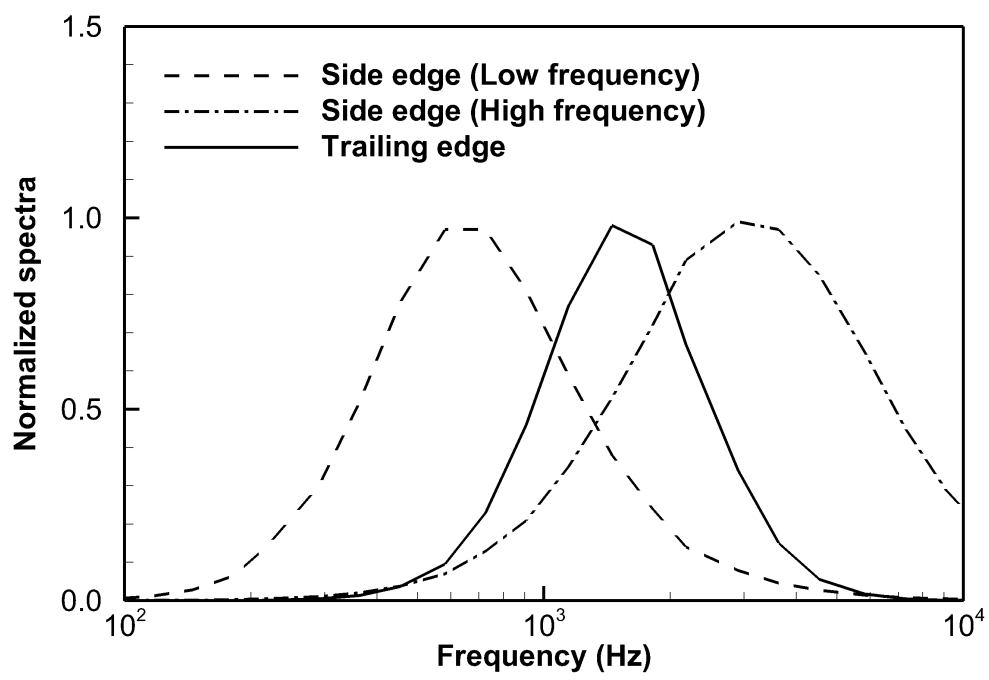

Fig. 2 Illustration of the normalized power spectral density.

$$
\beta=\beta_{0} e^{1.1 \sqrt{\delta}}
$$

and for the high frequency side-edge component

$$
\begin{cases}\beta=\beta_{0} e^{\delta / 4} & \delta<30^{\circ} \\ \beta=\beta_{0} e^{1+13 \delta / 60} & \delta \geqq 30^{\circ}\end{cases}
$$

Table 2 Energy conversion efficiency parameters.

\begin{tabular}{cccc}
\hline & For $P_{S L}$ & For $P_{S H}$ & For $P_{T}$ \\
\cline { 2 - 4 }$\beta_{0}$ & $1.25 \times 10^{-10}$ & $2.5 \times 10^{-10}$ & $1.25 \times 10^{-10}$ \\
\hline
\end{tabular}

where $\beta_{0}$ is an empirical constant. The values of $\beta_{0}$ are given in Table 2 for the three spectral components. Note that $\beta$ for the high frequency side-edge component is a piecewise function dependent on the deflection angle $\delta$. This function is used to represent the influence of the flap deflection on vortex rolling from the side-edges. At low deflection angles the vortices remain near the flap surface, whilst large flap deflections lead to an earlier lift-off of the vortices [13]. In order to discern the variation of high frequency noise due to flow feature variation, a deployment angle of $30^{\circ}$ is chosen as a separation point for the piecewise function (11). Figure 3 displays the increments of side-edge noise level as a 
function of deployment angle $\delta$; the increments are relative to the SPL at $\delta=20^{\circ}$. Note that the level of high frequency component increases quicker with $\delta$.

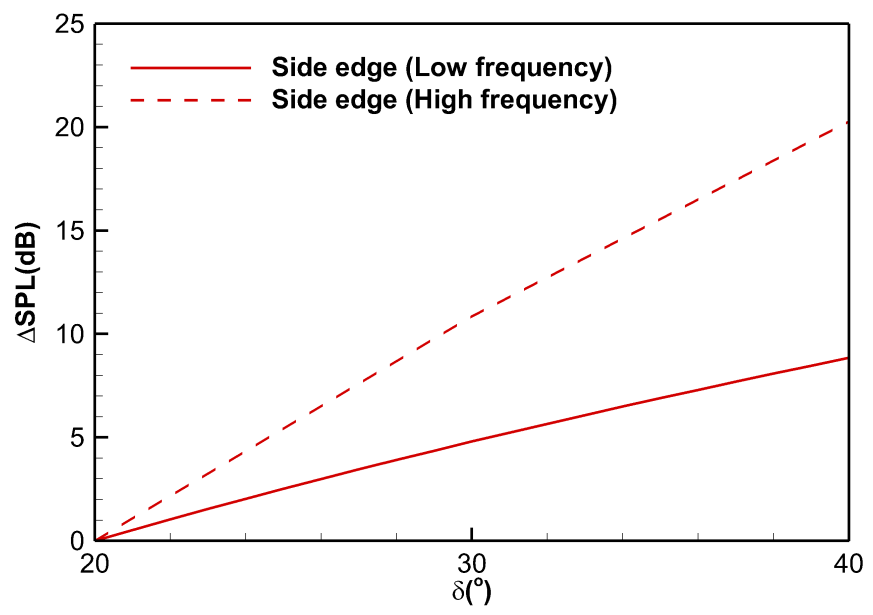

Fig. 3 Increment of side-edge noise due to deployment angle.

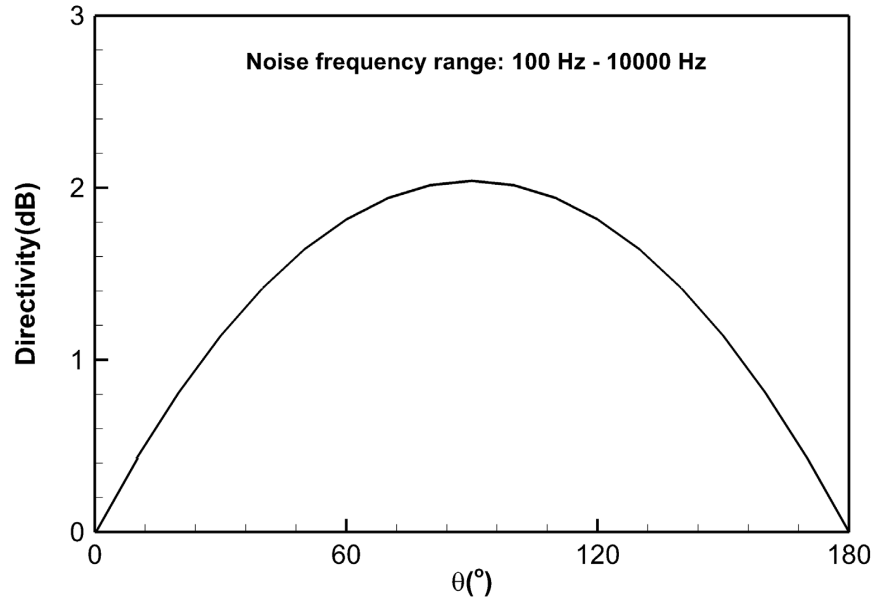

Fig. 4 Directivity factor for all the three spectral components.

\section{Directivity factor}

Due to the limited availability of experimental data, the directivity model will be presented only for emission angles in the flyover plane; on this plane the emission angle $\theta$ is measured from the downstream direction of the flight path, as illustrated in Figure 1 Test data have shown that the flap noise in the flyover plane has a directivity pattern varying only gradually with the emission angle [33]. The direction of maximum noise emission is roughly aligned with $\theta=90^{\circ}$ in the flyover plane, according to some experimental observations [34, 35]. Furthermore, in some experimental data [33], we noted that the difference is about $1 \mathrm{~dB}$ between the SPL at $\theta=90^{\circ}$ and at $\theta=30^{\circ}$. Thus, a directivity factor is proposed for all three components as

$$
D(\theta)=1+0.6 \sin \theta
$$

The directivity factor is shown in Figure 4 This factor is fitted from experimental data [33] in $30^{\circ}<\theta<150^{\circ}$. The applicability of this directivity factor needs further validation for the emission angles beyond the range of 
the experimental data. Moreover, the directivity factor is proposed from experimental data with frequencies 100 $\mathrm{Hz} \leq f \leq 10,000 \mathrm{~Hz}$.

\section{Numerical tests}

\section{A. A320-type flap}

The first case considered in our validation exercise is an isolated flap model - a nearly full-scale Airbus A320 geometry. This model has a chord length of $0.48 \mathrm{~m}$, and a span length $0.4 \mathrm{~m}$. The corresponding measurements were conducted by Rossignol et al. [13]. In this experiment, the distance between the flap model and the microphones was $0.6 \mathrm{~m}$.

In the experiment, the flap and the microphones were both fixed, therefore the predictions are performed with the convective amplification removed, $\Delta=1$. The flow conditions for the predictions are $c_{0}=340 \mathrm{~m} / \mathrm{s}$ and $\rho_{0}=1.18$ $\mathrm{kg} / \mathrm{m}^{3}$; no atmospheric absorption is included.

Figure 5 displays the predicted solutions for different deployment angles at $M=0.18$ and $\theta=90^{\circ}$ with the corresponding experimental data. The three components of the noise spectra are shown at all flap angles. It can be gleaned that the role of the high frequency component becomes increasingly important as the deployment angle is increased. This phenomenon can be explained by the fact that the flap noise at high deployment angles is dominated by the vortices from the side-edges. To validate the power law of Mach number dependence, Figure 9 shows the solutions for different Mach numbers with a deflection angle $25^{\circ}$. A good fit of the measured data is obtained with the power law $N_{S L}=N_{T}=5.5$ and $N_{S H}=6.5$.

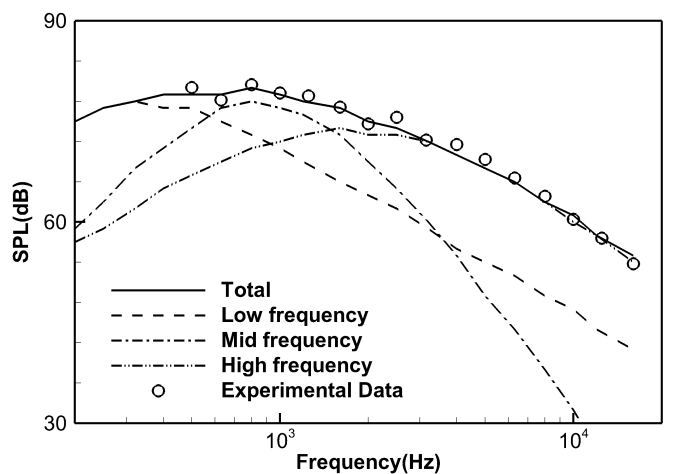

(a) $\delta=20^{\circ}$

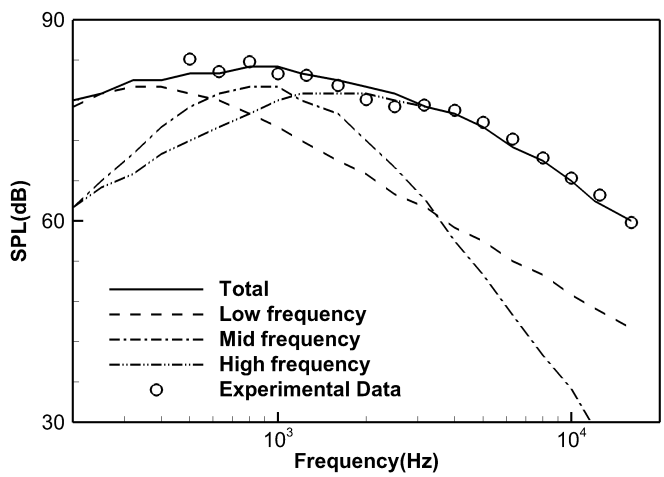

(b) $\delta=25^{\circ}$

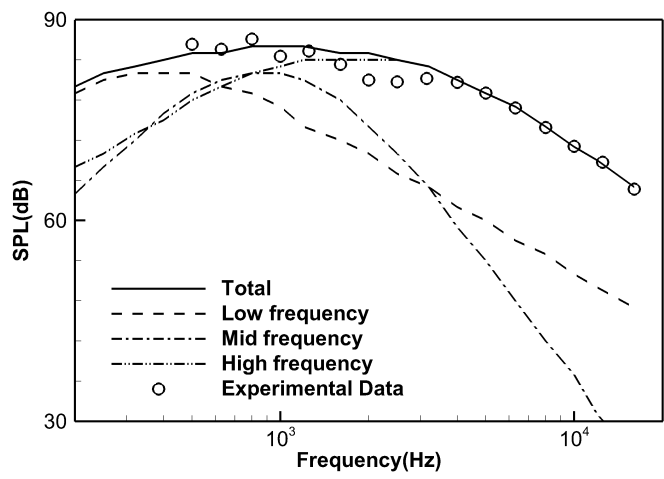

(c) $\delta=30^{\circ}$

Fig. 5 Theoretical predictions at increasing deployment angles at $\theta=90^{\circ}$ and $M=0.18$. Experimental data from Ref. [13]. 


\section{B. Fowler flap}

The second flap model considered is a half-span Fowler flap with a chord length of $0.12192 \mathrm{~m}$ and span 0.4572 $\mathrm{m}$. This model was tested in a quiet flow facility by Brooks et al. [11]. In that experiment, flaps with different edge geometry were measured. Since the present prediction model is proposed for a flat edge, we will only consider the corresponding experimental data. This flaps was further investigated by Hutcheson et al. [12] in the context of noise directivity. In all these experiments, the distance between the model and the microphones was $1.524 \mathrm{~m}$.

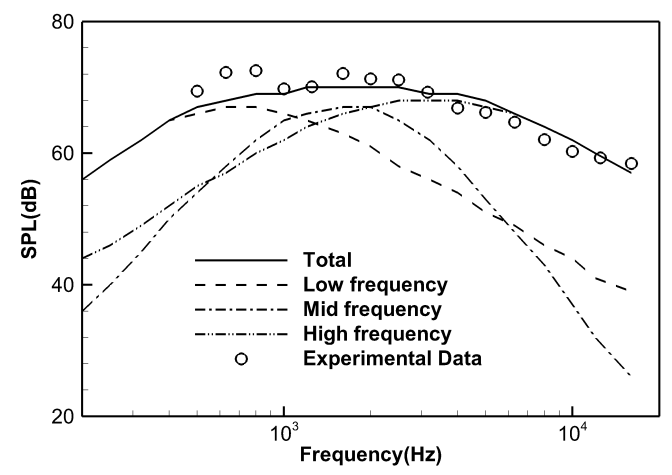

(a) $\delta=29^{\circ}$

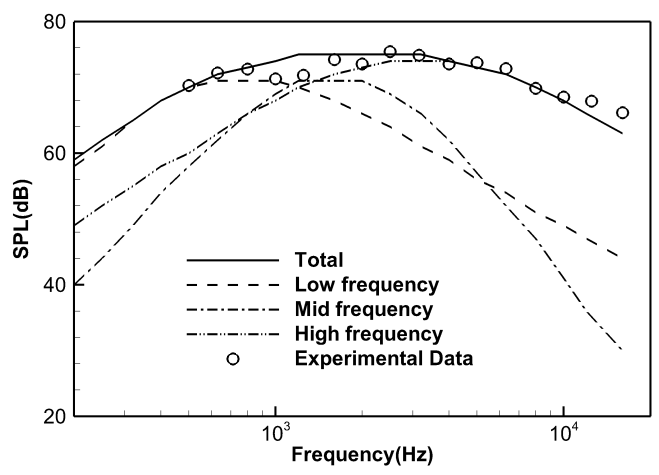

(b) $\delta=39^{\circ}$

Fig. 6 Noise predictions for Fowler flap at $\theta=90^{\circ}$; experimental data from Ref. [11].

Atmospheric absorption is ignored in this case, and the convective amplification is removed due to the fixed location of the flap and the microphones in the experiment. The flow conditions for the numerical results are $c_{0}=340 \mathrm{~m} / \mathrm{s}$ and $\rho_{0}=1.18 \mathrm{~kg} / \mathrm{m}^{3}$. Figure 6 shows the solutions for the deployment angles of $29^{\circ}$ and $39^{\circ}$, an emission angle $\theta=90^{\circ}$, and flow Mach number $M=0.17$. The predictions are in good agreement with experimental data. For the directivity factor, the numerical predictions are further compared with experimental data of Hutcheson et al. [12]. The comparison is carried out at four angles in the flyover plane, as shown in Figure 7 While most of the noise is very well captured by the model, the predicted solutions are rather inaccurate when the emission angle is $\theta=73^{\circ}$.

\section{Flap in high-lift wing model}

A flap model attached to a high-lift wing is chosen to test the capability of the prediction model, although the prediction model has been derived for an isolated flap. Aeroacoustic measurements were performed for a high-lift wing with a half-span flap by Rossignol [13], where the detailed geometry of the high-lift wing was described. This flap has the same profile geometry as the A320-type case in section III.A but with a chord length of $0.12 \mathrm{~m}$ and a 0.4 $\mathrm{m}$ span. To utilize the prediction model for this flap, the atmospheric absorption and the convective amplification are removed. Flow conditions for the predictions are $c_{0}=340 \mathrm{~m} / \mathrm{s}$ and $\rho_{0}=1.18 \mathrm{~kg} / \mathrm{m}^{3}$. For the present prediction, the distance between the flap model and the microphones is set to $0.6 \mathrm{~m}$.

To improve the agreement between experiments and prediction, an effective flap deployment angle is used to replace the nominal deflection $\delta$. This effective angle was also introduced by Rossignol [13] to empirically predict the flap noise. An effective deflection angle must be considered, because the influence of other elements of the high-lift wing system on the flow features of the flap.

Rossignol determined the effective angle based on linear extrapolation of the lift coefficient. The effective angles were about 5 to 6 degrees lower than the nominal flap deflection angle. In the same vein, we defined effective angles $17^{\circ}$ (corresponding to $\delta=25^{\circ}$ ) and $26^{\circ}$ (corresponding to $\delta=34^{\circ}$ ). Figure 8 shows the Mach number effect of the flap noise at $\delta=34^{\circ}$. An acceptable agreement is found between the predictions and experimental data, though not as good as the case shown in Figure 9

This behaviour indicates that the influence of other elements in the high-lift wing system on the flow feature of the flap have not been fully represented. The effect of deployment angle on the flap noise is demonstrated in Figure 10 at $M=0.15$. The prediction at $\delta=25^{\circ}$ is better than that at $\delta=34^{\circ}$. This result may be explained by the fact that the flow features of the flap are affected more significantly by other parts of the high-lift system at higher deployment angles. 


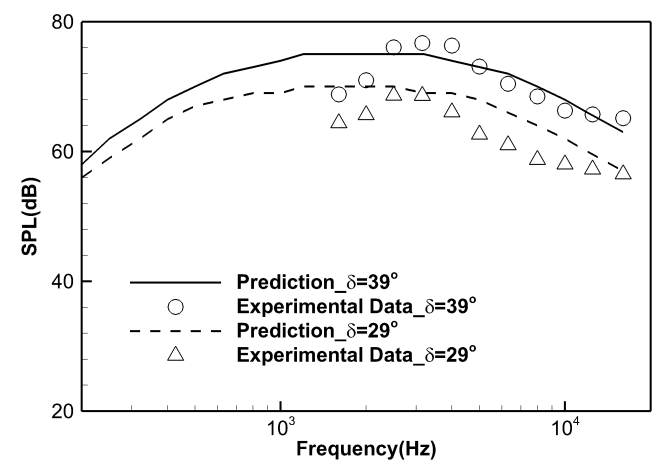

(a) $\theta=73^{\circ}$

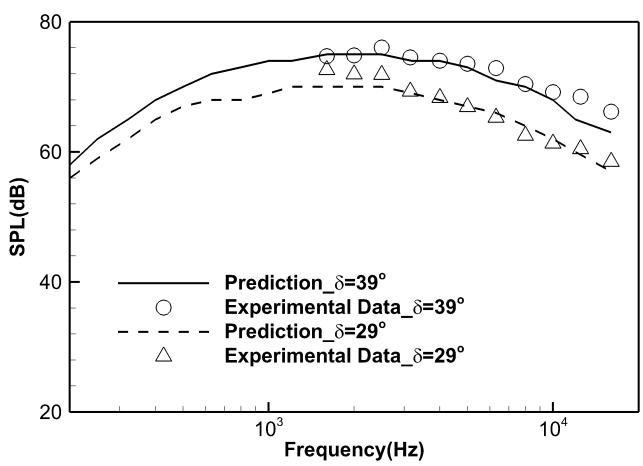

(c) $\theta=124^{\circ}$

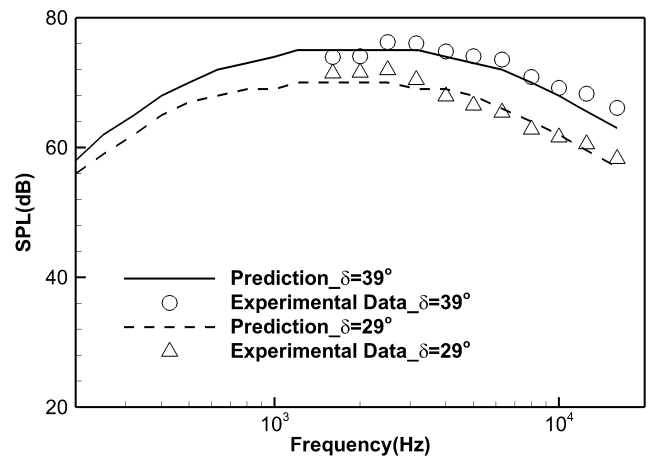

(b) $\theta=107^{\circ}$

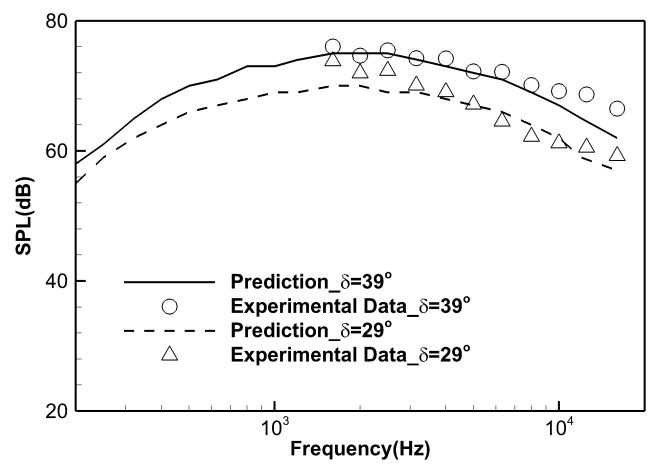

(d) $\theta=141^{\circ}$

Fig. 7 Acoustic directivity for deployment angles of $29^{\circ}$ and $39^{\circ}$; experimental data from Ref. [12].

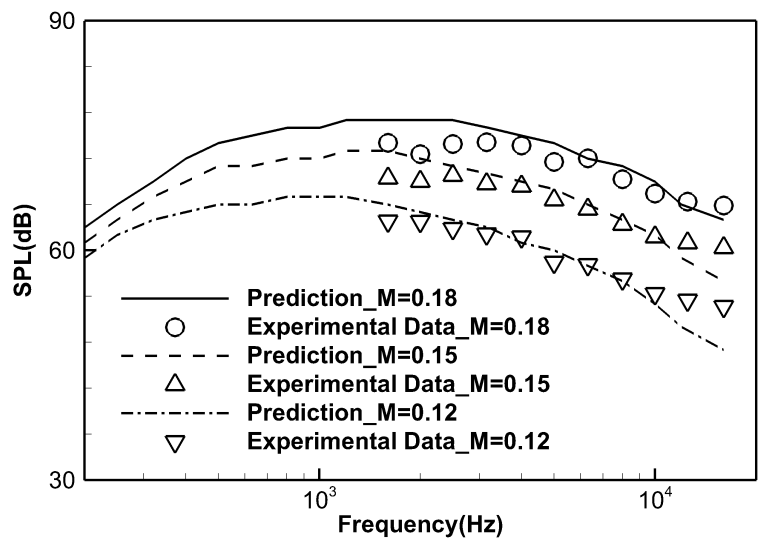

Fig. 8 Mach number effect in a high-lift wing at $\delta=34^{\circ}$; experimental data from Ref. [13].

\section{Conclusions}

A component-based model was proposed for flap noise prediction by decomposing the flap noise into the low frequency side-edge component, the high frequency side-edge component, and the trailing-edge component. At high angles of attack caused by deployed high-lift systems, the trailing-edge component in the flap noise is linked to the vortex shedding from trailing-edge. Supported by experimental data and theoretical analysis in the literature, the vortices rolling from side-edges are seen to be mainly responsible for the low- and the high frequency side-edge component.

The model presented in this paper has been used to predict noise radiated from various typical flap configurations, 


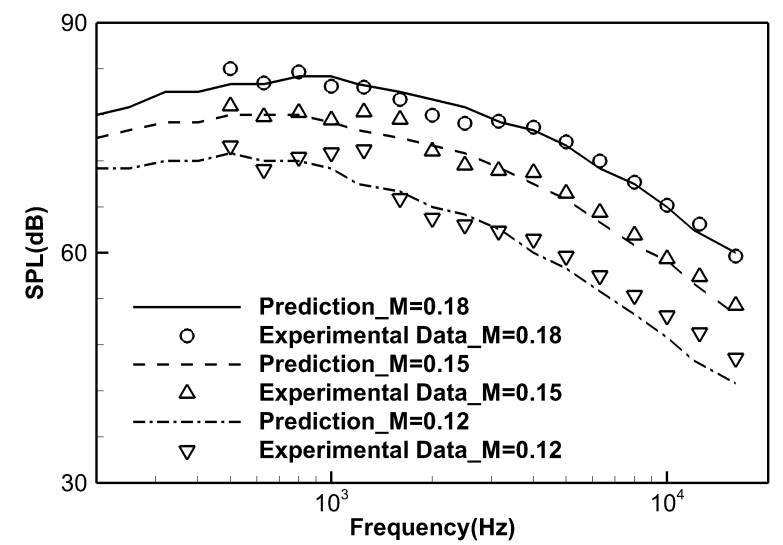

Fig. 9 Predictions for different Mach numbers at $\delta=25^{\circ}$; experimental data from Ref. [13].

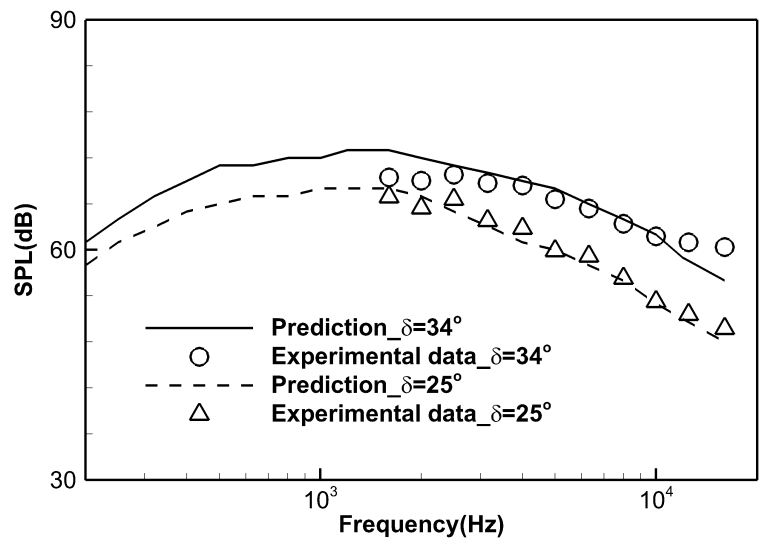

Fig. 10 Deflection effect at $M=0.15$; experimental data from Ref [13].

including an isolated A320-type flap, a half-span Fowler flap and a flap in a high-lift wing model. The predictions have shown a satisfactory agreement with the corresponding experimental data. The effect of Mach number and deployment angle on the flap noise has been well predicted. The directivity factor needs to be further validated since it was inferred from experimental data in $30^{\circ}<\theta<150^{\circ}$.

The noise model was proposed for a flap with a non-porous and blunt edge. The influence of installation effects on flow features needs further examination. More data are required to further investigate these effects, and only modifications of deployment angles were adopted for the flap in a high-lift wing case to account for them. A further line of investigation requires a more detailed exploration of the wing-flap interaction, and a better understanding of the effective flap deflection angle.

\section{Acknowledgments}

This study was supported by the National Key Research and Development Plan (Grant No. 2016YFB0200700) and the National Natural Science Foundation of China (Grant No. 11672321). Part of this research was also supported by EU Grant Agreement 277580 to the University of Manchester for integration into a comprehensive aircraft prediction model. 


\section{References}

[1] Dobrzynski, W. Almost 40 years of airframe noise research: what did we achieve?, Journal of Aircraft 47(2) (2010), pp. 353-367. DOI: $10.2514 / 1.44457$.

[2] Lele, S. K. and Nichols, J. W. A second golden age of aeroacoustics?, Philosophical Transactions of the Royal Society A 372 (2014) 20130321. DOI: 10.1098/rsta.2013.0321.

[3] Filippone, A. Aircraft noise prediction, Prog. Aero. Sci. 68 (2014), pp. 27-63. DOI: 10.1016/j.paerosci.2014.02.001.

[4] Fink, M. R. Noise component method for airframe noise, Journal of Aircraft 16(10) (1979), pp. 659-665. DOI: 10.2514/3.58586.

[5] Guo, Y. P., Yamamoto, K. J. and Stoker, R. W. Component-based empirical model for high-lift system noise prediction, Journal of Aircraft 40(5) (2003), pp. 914-922. DOI: 10.2514/2.6867.

[6] Lopes, L. V., Brentner, K. S. and Morris, P. J. Framework for a landing-gear model and acoustic prediction, Journal of Aircraft 47(3) (2010), pp. 763-774. DOI: 10.2514/1.36925.

[7] Streett, C. Numerical simulation of fluctuations leading to noise in a flap-edge flowfield, AIAA paper 1998-0628, 1998.

[8] Khorrami, M. R., Singer, B.A. and Radeztsky, R. H. Reynolds averaged Navier-Stokes computations of a flap side-edge flowfield, AIAA Journal 37 (1) (1999), pp. 14-22. DOI: 10.2514/2.687.

[9] Guo, Y., Joshi, M., Bent, P., and Yamamoto, K. Surface pressure fluctuations on aircraft flaps and their correlation with far-Field noise, Journal of fluid mechanics 415 (2000), pp. 175-202. DOI: 10.1017/S0022112000008740.

[10] Choudhari, M. M., Lockard, D. P., Macaraeg, M. G., Singer, B. A., Streett, C. L., Neubert, G. R., Stoker, R. W., Underbrink, J. R., Berkman, M. E., Khorrami, M. R. and Sadowski, S. S. Aeroacoustic experiments in the NASA Langley low-turbulence pressure tunnel, NASA/TM-2002-211432, 2002.

[11] Brooks, T. F. and Humphreys, W. M. Flap edge aeroacoustic measurements and predictions, Journal of Sound and Vibration 261 (2003), pp. 31-74. DOI: 10.1016/S0022-460X(02)00939-2.

[12] Hutcheson, F. V., Brooks, T. F. and Humphreys, W. M. Noise radiation from a continuous mold-line link flap configuration, AIAA paper 2008-2966, 2008.

[13] Rossignol, K. S. Flow field measurements to characterize flap side-edge noise generation, AIAA paper 2013-2061, 2013.

[14] Casalino, D., Hazir, A., Fares, E., Duda, B. and Khorrami, M. R. On the connection between flap side-edge noise and tip vortex dynamics, AIAA paper 2015-2992, 2015.

[15] Brooks, T. F. and Hodgson, T. H. Trailing edge noise prediction from measured surface pressures, Journal of Sound and Vibration 78 (1981), pp. 69-117. DOI: 10.1016/S0022-460X(81)80158-7.

[16] Brooks, T. F. and Schlinker, R. H. Progress in rotor broadband noise research, Vertica 7(4) (1983), pp. 287-307.

[17] Hutcheson, F. V. and Brooks, T. F. Effects of angle of attack and velocity on trailing edge noise determined using microphone array measurements, International Journal of Aeroacoustics 5(1) (2006), pp. 39-66. DOI: 10.1260/147547206775220425.

[18] Nakano, T., Fujisawa, N. and Lee, S. Measurement of tonal-noise characteristics and periodic flow structure around NACA0018 airfoil, Experiments in Fluids 40 (2006), pp. 482-490. DOI: 10.1007/s00348-005-0089-2.

[19] Sandberg, R., Jones, L., Sandham, N. and Joseph, P. Direct numerical simulations of tonal noise generated by laminar flow past airfoils, Journal of Sound and Vibration 320 (2009), pp. 838-858. DOI: 10.1016/j.jsv.2008.09.003.

[20] Kamruzzaman, M., Lutz, T., Herrig, A. and Krämer, E. Semi-empirical modeling of turbulent anisotropy for airfoil self-noise predictions, AIAA Journal 50(1) (2012), pp. 46-60. DOI: 10.2514/1.J050805.

[21] Chong, T. P., Josephb, P. F. and Kingan, M. J. An investigation of airfoil tonal noise at different Reynolds numbers and angles of attack, Applied Acoustics 74(1) (2013), pp. 38-48. DOI: 10.1016/j.apacoust.2012.05.016.

[22] Guo, Y. P. Aircraft flap side edge noise modeling and prediction, AIAA paper 2011-2731, 2011.

[23] Dobrzynski, W. and Buchholz, H. Full-scale noise testing on Airbus landing gears in the German Dutch Wind Tunnel, AIAA paper 97-1597, 1997.

[24] Guo, Y. P. Empirical prediction of aircraft landing gear noise, NASA/CR-2005-213780, 2005. 
[25] Lopes, L. V., Brentner, K. S., Morris, P. J., Lilley, G. and Lockhard, D. Complex landing gear noise prediction using a simple toolkit, AIAA paper 2005-1202, 2005.

[26] Lighthill, M. J. On sound generated aerodynamically I. General theory, Proceedings of the Royal Society of London A 211 (1952), pp. 564-587. DOI: 10.1098/rspa.1952.0060.

[27] Ffowcs Williams, J. E. and Hawkings, D. L. Sound generation by turbulence and surfaces in arbitrary motion, Philosophical Transactions of Royal Society of London A 264 (1969), pp. 321-342. DOI: 10.1098/rsta.1969.0031.

[28] Crighton, D. G. Basic principles of aerodynamic noise generation, Progress in Aerospace Sciences 16 (1) (1975), pp. $31-96$. DOI: 10.1016/0376-0421(75)90010-X.

[29] Heller, H. and Dobrzynski, W. Sound radiation from aircraft wheel-well/landing-gear configuration, Journal of Aircraft 14 (8) (1977), pp. 768-774. DOI: 10.2514/3.58851.

[30] Crighton, D. G., Dowling, A. P., Ffowcs Williams, J. E., Hekle, M. and Leppington, F. G. Modern methods in analytical acoustics, Springer, New York, 1992.

[31] Cozza, I. F., Iob, A. and Arina, R., Broadband trailing-edge noise prediction with a stochastic source model, Computers \& Fluids 57 (2012), pp. 98-109. DOI: 10.1016/j.compfluid.2011.12.011.

[32] Ewert, R., Appel, C., Dierke, J. and Herr, M., RANS/CAA based prediction of naca 0012 broadband trailing edge noise and experimental validation, AIAA Paper 2009-3269, 2009.

[33] Guo, Y. P. and Joshi, M. C. Noise characteristics of aircraft high lift systems, AIAA Journal 41(7) (2003), pp. 1247-1256. DOI: $10.2514 / 2.2093$.

[34] Rossignol, K. S. Development of an empirical prediction model for flap side-edge noise, AIAA paper 2010-3836, 2010.

[35] Miller, W. R., Meecham, W. C., and Ahtye, W. F. Large scale model measurements of airframe noise using cross-correlation techniques, J. Acoust. Soc. Am. 71(3) (1982), pp. 591-599. DOI:10.1121/1.387530. 